\title{
Health problems due to unsafe sex among youths: condom use negotiation and consistent use, one way to address them
}

\begin{abstract}
Condoms prevent the spread of STIs if used correctly and consistently, and have proved to reduce the risk of unintended pregnancies. About $79 \%$ of women and $83 \%$ of men know the preventive efficacy of condoms. Whereas the level of condom knowledge and approval among adolescents is high, the level of actual use among the sexually active is low. In Uganda, the burden of health problems due to unsafe sex including sexually transmitted diseases, unplanned early pregnancies and related complications among others is still high among youths and it poses more negative impacts on females than males. Thus knowledge is of trivial value if people, especially those in most need of the protection, feel timid to claim for what they know as right. This raises need to embark on women's involvement in protecting them against this burden through empowering them to negotiate for safe sex practices with their sexual partners.
\end{abstract}

Volume I Issue I - 2014

\author{
Ndagire Regina Nabikindu \\ Department of Nursing, Makerere University College of Health \\ Sciences, Uganda
}

\section{Correspondence: Ndagire Regina Nabikindu, Department of Nursing, Makerere University College of Health Sciences, Uganda,Tel 078I015336,Email ndaginar@gmail.com}

Received: November 05, 2014 | Published: November 07,2014

Keywords: negotiation, HIV/AIDs, condom, pregnancy, adolescents, youths, young adults

Abbreviations: STIs, sexually transmitted infections; HIV, human immune virus; AIDS, acquired immune disease

\section{Introduction}

Efforts have been made to abolish the tradition of early marriages, but these have lengthened the period spent unmarried yet sexually active which is life threatening. In Uganda, the age of 15 has been identified as the mean age of initiation of sexual intercourse among young adults. ${ }^{1}$ Nationally, 21\% of women aged 15-24 are unmarried but sexually experienced; that is, they have ever had sexual intercourse. ${ }^{2}$ The high rates of unsafe early sexual activity and the resultant pregnancies among young adults have significantly contributed to Uganda's high fertility rate. The Total Fertility Rate of Uganda is 6.2 births per woman while the fertility rate of 15-19 and 20-24 age groups is 134 and 313 per 1,000 women respectively. ${ }^{1}$

The burden of health problems related to unsafe sex practices is still alarmingly high in Uganda. To begin with, in most high-burden countries including Uganda, there is still a high prevalence of STIs with twice as many new HIV infections in the adolescent age group compared with the deaths; therefore, in these countries, the epidemic in adolescents is still growing.,

Gender inequality has also been established in the HIV/AIDS burden in sub-Saharan Africa ${ }^{5,6}$ this highlights need for genderoriented efforts in eradicating the pandemic. Statistics show that in 2007, of all the HIV positive adults in sub-Saharan Africa, 61\% were women and $75 \%$ of young people infected were girls. ${ }^{7}$ Apparently, the prevalence of HIV among young people aged $15-24$ is $4.0 \%$ in females, ${ }^{8}$ which is almost double that of their male counterparts $2.3 \%$.

While the risk of contracting STIs for both genders during unprotected sex might be equal, the pregnancy risk and the related probable physiological and socio-economic complications, including dropping out of school, being neglected by family, social misfit, maternal injury, and death are faced only by the females. Uganda has the largest teenage pregnancy rate in sub-Saharan Africa, with half of its girls giving birth before the age of $18 .{ }^{9}$ The Uganda Demographic and Health Survey - 2006 indicates that, one in every four teenagers is pregnant. This represents about $25 \%$ of the teenagers getting pregnant annually. More than four in 10 births $(43 \%)$ are unplanned. ${ }^{1}$ Some of these give birth to healthy children, but many experience adverse effects of unplanned early pregnancies and child birth like fistulas, unsafe abortions, and death hence contributing to the high maternal mortality rate in Uganda which currently is recorded at 438 deaths per 100,000 live births. About $17.6 \%$ and $22.6 \%$ of these occur among 15-19 and 10-24 age groups respectively.

\section{Conclusion}

\section{Where we are and the way forward}

Nevertheless, a condom is one proven contraceptive with efficacy to control all these health problems and hence the related socioeconomic problems. Condoms have been proved to guarantee more than $90 \%$ effectiveness at prevention of STDs if used correctly and consistently. ${ }^{10}$ And as a contraceptive method, a condom is known to reduce the risk of unintended pregnancies, with the effectiveness estimated at $85-98 \%$ and $79-95 \%$ for male and female condoms respectively. ${ }^{11}$

On that note, efforts have been made to increase condom uptake by youths among which there is health education and demonstrations, and availing free condoms, scientific studies to embark on condom utilization to mention but a few. But attention has been focused more on the male involvement. Culture has also portrayed it as a male's role to initiate sex including safe sex. Despite all that, it is evident that health problems due to unsafe sex practices are still on the rise among adolescents affecting the females more than their male counterparts. This emphasizes the need to also embark on female involvement in safe sex practices that is negotiating for condom use and ensuring consistent condom use. 
But one needs self-confidence to negotiate for these practices, a behavior that is fundamental today when the burden of health problems due to unsafe sex is despicable. According to the UDHS 2011, 79\% of women and $83 \%$ of men know the preventive efficacy of condoms; however the prevalence of condom use among the 15-24year olds is $47.3 \%$, which is still low for prevention of STIs, pregnancies, and other related problems. ${ }^{1}$ This indicates that knowledge alone cannot increase condom use uptake and is of trivial value if people, especially the girls in most need of the protection, feel powerless to influence their partners into the actual use of condoms.

Because females are more affected than males by the repercussions of unsafe sex practices, their involvement in safer sex practices is equally important and needs to be accentuated. There is need to emphasize their position in protecting themselves against this burden. Unfortunately, in Uganda, females' involvement in safer sex practices has not been given the due attention. Women hardly know their role and those who know do not play their part. Review of literature shows that neither have any studies been done concerning condom use negotiation in Uganda nor are interventions in this regard.

\section{Recommendation}

According to a study by Perrino and colleagues, people who in addition to communication can persuade their partners to use condoms are more likely to actually use them than those who cannot. ${ }^{12}$ Upon such evidence, efforts need to be engineered towards encouraging young females to be actively involved in safe sex practices and among these is empowering them to negotiate for condom use with their sexual partners and consistently using them. This empowerment could be through education, equipping them with negotiation skills including personal defense/ protective skills, support groups and organizations, and socio-economic support among others. They need to be encouraged and supported not to go in for unprotected sex. And as such the burden of health problems among females due to unsafe sex will be reduced.

\section{Acknowledgements}

None.

\section{Conflict of interest}

The author declares no conflict of interest.

\section{References}

1. Uganda Bureau of Statistics and ICF International. Uganda Demographic and Health Survey 2011. USA: UBOS, ICF International; 2012.

2. Singh S, Prada E, Mirembe F, et al. The incidence of induced abortion in Uganda. Int Fam Plan Perspect. 2005;31(4):183-191.

3. Kasedde S, Kapogiannis BG, McClure C, et al. Executive Summary: Opportunities for Action and Impact to Address HIV and AIDS in Adolescents. J Acquir Immune Defici Syndr. 2014;66(Suppl2):S139-S143.

4. United Nations Children's Fund. Towards an AIDS-free GenerationChildren and AIDS: Sixth Stocktaking Report. USA: UNICEF; 2013.

5. Anarfi JK. Vulnerability to sexually transmitted disease: street children in Accra. Health Transit Rev. 1997;7(Suppl):281-306.

6. Oppong JR. A vulnerability interpretation of the geography of HIV/AIDS in Ghana, 1986-1995. The Professional Geographer. 1998;50(4):437-448.

7. UNAIDS/WHO. AIDS epidemic update. Geneva, Switzerland; 2007.

8. UBOS. Uganda: statistics. 2013.

9. UNFPA. State of the world population 2003. USA; 2003.

10. Weller S, Davis K. Condom effectiveness in reducing heterosexual HIV transmission. Cochrane Database Syst Rev. 2002;1(1):CD003255.

11. CDC. Unintended Pregnancy Prevention: Contraception. 2013.

12. Perrino T, Fernandez MI, Bowen GS, et al. Low-income African American women's attempts to convince their main partner to use condoms. Cultur Divers Ethnic Minor Psychol. 2006;12(1):70-83. 